Document downloaded from:

http://hdl.handle.net/10251/56283

This paper must be cited as:

David Gomez-Barquero; Gozálvez Serrano, D.; Gómez Molina, PF.; Cardona Marcet, N. (2011). Fading Margin Reduction due to Inter-Burst Upper Layer FEC in Terrestrial Mobile Broadcast Systems. IEEE Transactions on Vehicular Technology. 60(7):3110-3117. doi:10.1109/TVT.2011.2162535.

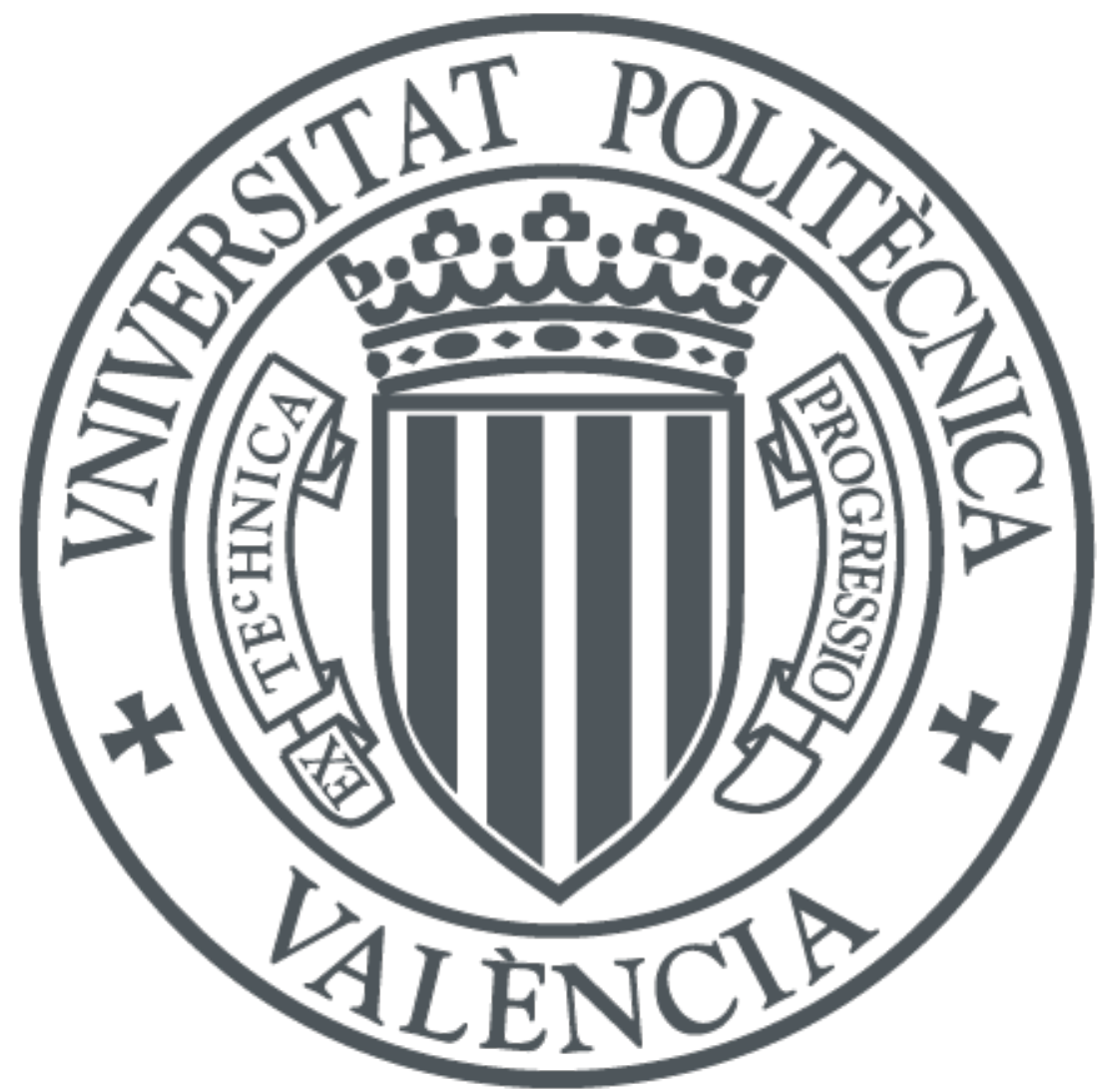

The final publication is available at

http://dx.doi.org/ 10.1109/TVT.2011.2162535

Copyright Institute of Electrical and Electronics Engineers (IEEE)

Additional Information 


\title{
Fading Margin Reduction due to Inter-Burst Upper Layer FEC in Terrestrial Mobile Broadcast Systems
}

\author{
David Gómez-Barquero, David Gozálvez, Pedro F. Gómez, Narcís Cardona
}

\begin{abstract}
In this letter we investigate the reduction of the shadowing fading margin that can be achieved with inter-burst Upper Layer Forward Error Correction (UL-FEC) in terrestrial mobile broadcast systems with time-slicing (i.e., discontinuous transmission). A theoretical framework is derived, for both streaming and file delivery services, as a function of the number of bursts jointly encoded, the UL-FEC code rate, the shadowing standard deviation, and the ratio between the moved distance by the user during the cycle time between bursts and the shadowing correlation distance. Results are validated with DVB-H and DVB-SH laboratory measurements.
\end{abstract}

Index Terms-Mobile TV, DVB-H, DVB-SH, DVB-NGH, inter-burst FEC, UL-FEC, MPE-iFEC, AL-FEC.

\section{INTRODUCTION}

$\mathbf{I}^{\mathrm{r}}$ $\mathrm{N}$ wireless communications mobile users typically experience signal fading effects due to the superposition of multiple propagation paths between transmitter and receiver. In general, it can be distinguished between slow or long-term fading due to local shadowing effects, commonly known as shadowing, and fast or short-term fading due to the coherent superposition of the signals reaching the terminal, commonly known as fast or multipath fading [1]. Shadowing implies significant variations of several $\mathrm{dB}$ over distances of several tens of meters (comparable to the widths of buildings in the region of the mobile), whereas fast fading involves variations on the scale of a half-wavelength and frequently introduces variations as large as 35 to $40 \mathrm{~dB}$.

Signal fading can be compensated with forward error correction (FEC) with a time interleaving large enough to essentially average out the fading statistics. FEC mechanisms rely on the transmission of redundant repair information (parity data) such that the receiver can correct errors occurred during the transmission. For the FEC code to be efficient, the different code symbols should ideally experience uncorrelated fading. In this case, it is possible to cope with erasure rates as large as the inverse of the code rate. Otherwise, the efficiency of the FEC mechanism is reduced.

In case of fast fading, a relatively short time interleaving is usually required to eliminate it (in the order of few to several milliseconds, depending on the user velocity and operating

\footnotetext{
Manuscript received March 23, 2011; revised June 16, 2011. This work was partially supported by the Spanish Ministry of Industry, Tourism and Commerce under the Celtic project Enabling Next Generation Networks for Broadcast Services ENGINES (TSI-020400-2010-108).

The authors are with the iTEAM Research Institute of the Universidad Politécnica de Valencia (UPV), Camino de Vera s/n, Edificio 8G, 46022, Valencia, Spain (e-mail: \{dagobar, dagoser, pedgomo1, ncardona\}@iteam.upv.es).
}

frequency). This is traditionally done at the physical layer as part of the radio interface for wireless communication systems.

This is typically not a feasible way to cope with shadowing due to memory and latency constraints. The memory requirement is directly proportional to the service data rate, the interleaving duration, the proportion of parity data transmitted, and the interleaver profile. On the other hand, terminals should in principle wait and store all data jointly encoded before decoding. The traditional approach to cope with shadowing consists in adding an extra fading margin of several $\mathrm{dB}$ to the link budget, in order to assure a reliable communication with a given probability at the cell edge. Nevertheless, for mobile users it is in principle possible to reduce the required fading margin exploiting the time diversity of the mobile channel using a FEC scheme spanning a sufficiently long time. Recent advances in FEC make this possibility a reality.

Today, newly deployed mobile digital video broadcasting standards such as DVB-H (Handheld) [2] and DVB-SH (Satellite to Handhelds) [3], define FEC schemes with long time interleaving profiles to exploit the time diversity of the mobile channel ${ }^{1}$. These mobile broadcasting technologies are characterized by a discontinuous transmission technique, known as time-slicing, where data is periodically transmitted in so-called bursts. Hence, these FEC schemes are usually referred to as inter-burst FEC. For file download services, both DVB-H and DVB-SH adopt application layer FEC (AL-FEC) using Raptor coding [5]. For streaming services, in DVB-SH it is possible to provide long time interleaving together with fast zapping support either at the physical layer or at the link layer. The physical layer FEC outperform the link layer FEC at the expense of higher memory requirements [6]. The link layer FEC mechanism is known as multi protocol encapsulation inter-burst FEC (MPE-iFEC) [7], and it specifies two coding schemes. One mandatory using sliding ReedSolomon encoding (SRSE), and another optional using Raptor coding. It should be pointed out that such mechanisms can be also applied in DVB-H [8].

In this paper, we derive a theoretical framework to evaluate the shadowing fading margin reduction that can be achieved in a generic terrestrial broadcast system with inter-burst upper layer FEC (UL-FEC, i.e. either link or application layer [9]). Numerical results are validated with DVB-H/SH figures. Physical layer FEC is out of the scope of the paper. Interested readers are referred to the DVB-SH implementation guidelines [6] for performance comparison details with link layer FEC.

\footnotetext{
${ }^{1}$ Such FEC schemes are currently also under consideration in the standardization process of the next generation handheld DVB-NGH standard [4].
} 
The rest of the paper is structured as follows. Section II describes and formulates the problem. It explains how the shadowing fading margin is computed, and illustrates the potential gain that can be achieved with inter-burst FEC. Section III presents a theoretical framework to compute the fading margin reduction for streaming delivery with interburst FEC for the case of uncorrelated shadowing. Section IV extends the framework presented in Section III for the more generic case of correlated shadowing. In Section V, the theoretical results are validated with DVB-H/SH laboratory measurements. Section VI discusses how to apply the proposed framework for file download services. Finally, we give some concluding remarks in Section VII.

\section{PRoblem Description}

We consider a generic terrestrial mobile broadcast system with time-slicing, where the different services are transmitted at the same frequency but in different time slots (bursts). Without loss of generality, we assume that the cycle time between bursts of the same service is constant. The system is characterized by a receiver sensitivity, which is the minimum signal level at which reception is possible. Fast fading is assumed to be eliminated at the physical layer with some form of FEC and short time interleaving during the burst duration. Shadowing is assumed to remain constant during this time. It is worth to note that these assumptions are commonly accepted in DVB-H and DVB-SH. Their physical layer FEC have a brick-wall characteristic that corrects above a certain signalto-noise ratio (SNR) threshold whereas fails below; and the burst duration is in the order of few hundreds of milliseconds.

The variations of the shadowing in $\mathrm{dB}$ are described by a zero-mean Gaussian random variable with standard deviation $\sigma_{l}$ and correlation distance $d_{c o r r}$ [1]. The shadowing standard deviation is known as the location variability, and it determines the spread of the signal field strength around the mean value. Values typically used for terrestrial outdoor broadcasting signals are $5.5 \mathrm{~dB}$ in the UHF band and $8 \mathrm{~dB}$ in the $\mathrm{S}$ band [10]. On the other hand, the spatial correlation is usually modeled as a first-order exponential model [11]:

$$
\rho(d)=e^{-d / d_{\text {corr }}},
$$

where $d_{\text {corr }}$ is distance taken for the autocorrelation to fall by $e^{-1}$. Typical values range from $20 \mathrm{~m}$ to $100 \mathrm{~m}$, depending on the environment surrounding the receiver [1].

Shadowing makes coverage prediction statistical, such that what is predicted is the signal availability rather than the signal level, see Fig. 1. The outage probability at a single reception point with an average signal level $\overline{S N R}$, is equal to the probability that the shadowing increases the mean path loss by at least $\overline{S N R}-S N R_{t h}$ (in $\mathrm{dB}$ ), where $S N R_{t h}$ is the required SNR threshold for the system. The outage probability can be computed as:

$$
\text { Outage Probability }=Q\left(\frac{\overline{S N R}-S N R_{t h}}{\sigma_{l}}\right),
$$

where the $Q(\cdot)$ function is the complementary cumulative normal distribution:

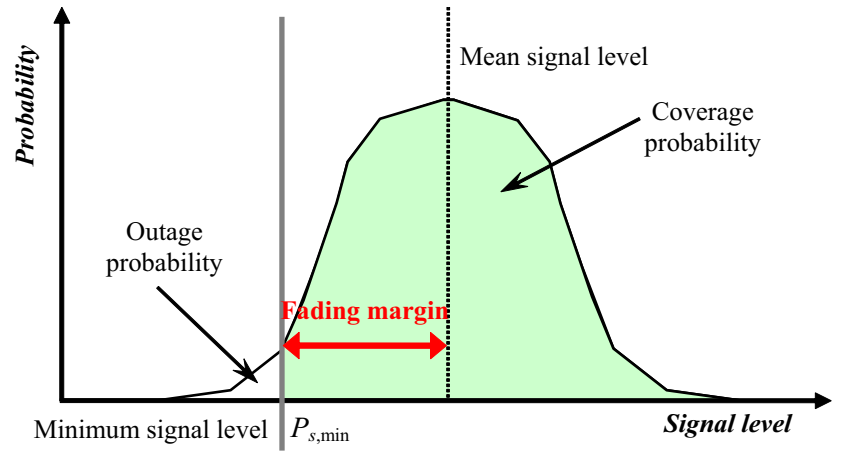

Fig. 1. Shadowing fading margin concept.

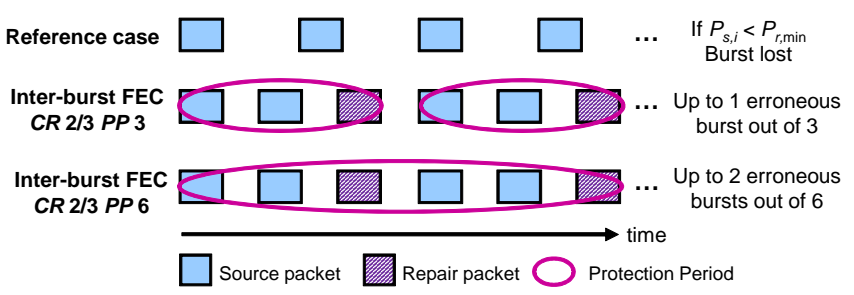

Fig. 2. Inter-burst UL-FEC concept in a generic mobile broadcast system with time-slicing. Under our assumptions, if the received signal strength is lower than the receiver sensitivity the burst (slot) is lost.

$$
Q(x)=\frac{1}{\sqrt{2 \pi}} \int_{x}^{\infty} e^{-t / 2} d t .
$$

The shadowing fading margin required to assure a reliable communication with a given probability, also known as correction location factor in broadcasting $C_{l}$ [10], is usually expressed as the product of the shadowing standard deviation, $\sigma_{l}$, and a correction coefficient, $\mu$ :

$$
C_{l}=\mu \cdot \sigma_{l} .
$$

The value of the correction coefficient $\mu$ depends only on the target coverage probability. It takes the value of 0 for $50 \%, 0.52$ for $70 \%, 1.28$ for $90 \%, 1.64$ for $95 \%$, and 2.33 for $99 \%$ [10]. Typical coverage probability targets in DVB-H and DVB-SH (terrestrial) are $95 \%$ for good pedestrian reception and $99 \%$ for good vehicular reception.

We consider an ideal systematic FEC erasure code operating above the physical layer that produces repair packets which are sent along with the original source packets to compensate for potential losses due to shadowing ${ }^{2}$. The original multimedia stream is divided into source blocks, which are treated and encoded independently. The inter-burst UL-FEC configuration parameters are: the ratio of source information to the total amount of source and repair data transmitted, commonly known as code rate $(C R)$, and the number of bursts jointly encoded, here denoted as protection period $(P P)$. An illustrative example is depicted in Fig. 2.

\footnotetext{
${ }^{2}$ The standardized UL-FEC codes in DVB-H and DVB-SH are ReedSolomon and Raptor codes [9]. Reed-Solomon codes have an optimal erasure recovery capacity. They can correct as many lost packets (erasures) as the number of parity packets transmitted. Raptor codes achieve very close to ideal performance. They require only slightly more packets than the number of source packets (e.g. $1 \%$ reception overhead)
} 


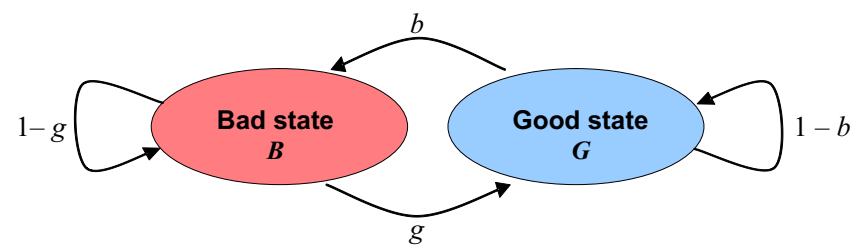

Fig. 3. The Gilbert-Elliott channel.

The error correction capability of inter-burst UL-FEC in terms of percentage of erroneous bursts that can be corrected within each protection period depends only on the code rate. The more repair data is transmitted, the more robust the transmission (at the expense of a decreased system capacity). However, the transmission robustness depends also on the protection period. In general, the longer the interleaving depth, the more robust the transmission (at the expense of an increased latency and larger memory capabilities at the terminals). In practice, depending on the channel, the reception speed, and the code rate, there is a point where no visible gain is achieved by increasing the interleaving.

In the example shown in Fig. 2, a code rate 2/3 can correct up to $33 \%$ errors, and thus it will be possible to recover from one erroneous burst out of three (for $P P$ 3), two erroneous bursts out of six (for $P P$ 6) and so on; as soon as all other bursts within the protection period are correctly received.

Inter-burst UL-FEC reduces the fading margin required to cope with shadowing because the probability of correctly receiving a burst, henceforth denoted as service availability, is higher than the coverage probability (percentage of covered locations with an average signal level higher than the receiver sensitivity). This gain stems from the time diversity of the mobile channel due to the mobility of the users. Therefore, for a given scenario and transmission configuration (code rate and protection period), the gain depends on the user trajectory and velocity [12].

In this case, the analysis can be easily derived analytically using the well-known Gilbert-Elliott (GE) channel model, see Fig. 3. The GE model dates back to the early 60's [13]-[14]. In this model, the channel is assumed to be either in a good state where the error rate is small, or in a bad state where the error rate is large. The good and the bad states are denoted $G$ and $B$ respectively, and the probabilities that the channel changes from the good state to the bad state and vice versa $b$ and $g$. We further assume that the probability of erasure is 1 in the bad state and 0 in the good state. The stationary probabilities that the channel is in the good state, $P^{\infty}(G)$, and in the bad state, $P^{\infty}(B)$, are:

$$
P^{\infty}(G)=\frac{g}{g+b} ; P^{\infty}(B)=\frac{b}{g+b} .
$$

\section{Theoretical Analysis for Streaming Delivery WITH UNCORRELATED SHADOWING}

\section{A. Analytical Framework}

The maximum reduction of the shadowing fading margin occurs when there is no shadowing correlation between reception conditions of consecutive bursts. In this case, the GE

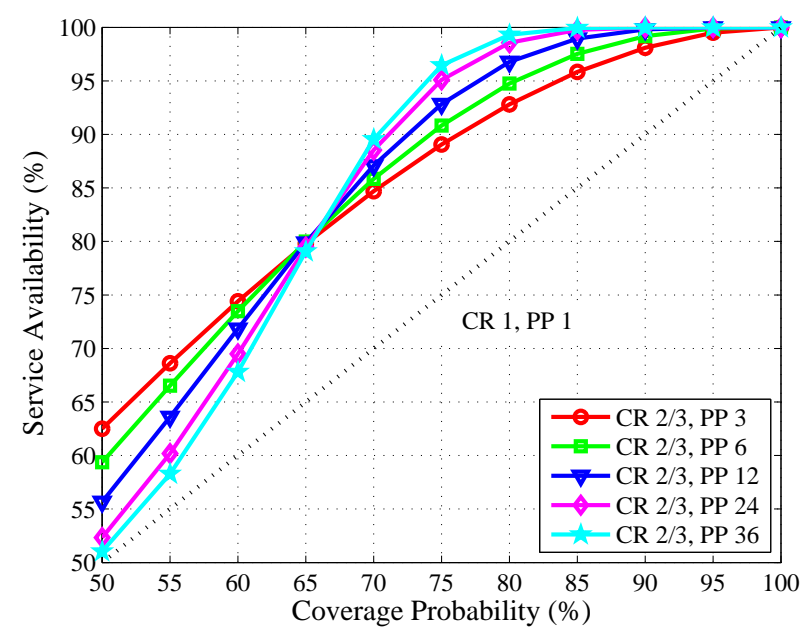

Fig. 4. Service availability vs. Coverage probability. Uncorrelated shadowing.

channel is memoryless, and the probability of being in the good or bad state is independent of the previous state. The GE channel becomes memoryless when $g+b=1$. Therefore, $g$ and $b$ are the coverage and outage probabilities respectively.

Under these conditions, if the GE channel is observed at $n$ consecutive instants of time, the probability that the channel is in the bad state $d$ times $(0 \leq d \leq n)$, denoted as $\mathrm{P}_{n}(d)$, is given by:

$$
\mathrm{P}_{n}(d)=\left(\begin{array}{l}
n \\
d
\end{array}\right) \cdot g^{(n-d)} \cdot(1-g)^{d} .
$$

With inter-burst UL-FEC, given the code rate, $C R$, and the protection period $P P$, the minimum number of bursts needed to decode a source data block, $n_{\min }$, can be computed as:

$$
n_{\text {min }}=\lceil C R \cdot P P\rceil,
$$

where $\lceil\cdot\rceil$ is the ceiling function.

Finally, the service availability, $S_{a v}$, defined as the probability of receiving a burst correctly after inter-burst UL-FEC decoding, can be computed using Eq. (6) as:

$$
S_{a v}=1-\sum_{i=P P-n_{m i n}+1}^{P P} \mathrm{P}_{P P}(i) \cdot\left(\frac{i}{P P}\right) .
$$

Note that the second term in Eq. (8) covers all the cases where the inter-burst UL-FEC decoding is not successful.

\section{B. Analytical Results}

Fig. 4 shows the service availability as a function of the coverage probability for inter-burst UL-FEC with code rate $2 / 3$ for different protection periods. The case without UL-FEC (CR 1, PP 1) is also shown for comparison. In this case, the service availability is equal to the coverage probability.

In the figure we can see how the service availability perceived by the users with inter-burst UL-FEC is considerably increased compared to the reference case, and that the improvement depends on the protection period and the coverage probability. It should be noted that increasing the interleaving depth (protection period) is only beneficial when the code rate 


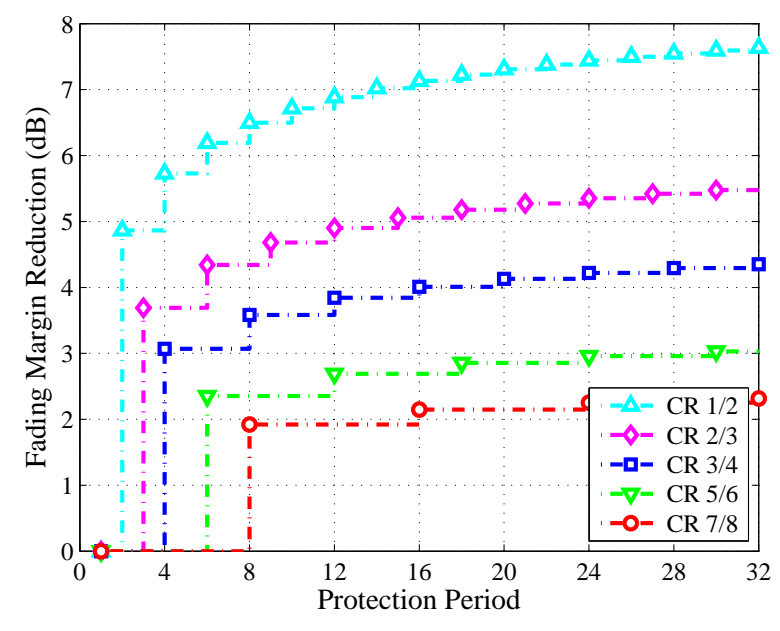

Fig. 5. Shadowing fading margin reduction for $95 \%$ service availability vs. Protection period in burst units. Uncorrelated shadowing: $\sigma_{l}=5.5 \mathrm{~dB}$, $d_{\text {corr }}=0 \mathrm{~m}$.

is robust enough to correct the total number of errors (i.e., for coverage probabilities above $66 \%$ in the figure). Otherwise, the performance is reduced.

From the results shown in Fig. 4, it can be obtained a new distribution factor $\mu$ for computing the fading margin as in Eq. (4). Therefore, the fading margin reduction depends on the code rate, protection period and target service availability, and it is directly proportional to the standard deviation of the shadowing.

Fig. 5 presents the fading margin reduction as a function of the protection period for different UL-FEC code rates $\left(\sigma_{l}\right.$ is $5.5 \mathrm{~dB}$ and the service availability $95 \%$ ). In this case, the fading margin without inter-burst UL-FEC would be $9 \mathrm{~dB}$. We can see that the reduction increases for more robust code rates and for larger protection periods. The potential gain is very significant, even for short interleaving depths, especially for code rates $1 / 2$ and $2 / 3$ (almost $5 \mathrm{~dB}$ and $4 \mathrm{~dB}$ reduction for protection periods of jut 2 and 3 bursts, respectively). Finally, we want to point out that the reduction increases not only for larger values of the standard deviation of the shadowing (e.g., for $\sigma_{l} 8 \mathrm{~dB}$ reductions are $45 \%$ larger than for $5.5 \mathrm{~dB}$ ), but also for more demanding service probabilities. For example, for $99 \%$ service availability the reduction is between $1 \mathrm{~dB}$ and $3 \mathrm{~dB}$ larger than the values shown in Fig. 5.

\section{Theoretical Analysis for Streaming DeliVery WITH CORRELATED SHADOWING}

The shadowing correlation between consecutive bursts depends on the shadowing correlation distance, the cycle time between bursts, and the user velocity, see Eq. (1). In general, the assumption that the shadowing is uncorrelated between consecutive bursts does not apply in most cases. Typical cycle time values for mobile TV streaming services in DVB-H/SH are in the range of one to few seconds. Therefore, only users with very high speeds experience uncorrelated shadowing between consecutive bursts, and the fading margin reduction is, in practice, smaller than the values presented in the previous section. The gain decreases for larger shadowing correlation distances, smaller user velocities, and cycle time values. Nevertheless, it should be clear that the maximum reduction does not depend on these parameters. These parameters basically determine the level of time interleaving depth required to achieve a target shadowing fading margin. Indeed, all three parameters can be combined into one: the ratio between the moved distance by the user during the cycle time and the shadowing correlation distance.

\section{A. Analytical Framework}

In case there is correlation between consecutive burst, the GE channel is no longer memoryless, and the probability of being in the good or the bad state depends on the previous state (i.e., $g+b \neq 1$ ). The parameters of the GE channel can be obtained taking into account that the GE has an exponential correlation function like the shadowing [15]. At time $m$, the correlation function of the GE channel, $\rho(m)$, equals to:

$$
\rho(m)=(1-b-g)^{m}
$$

Comparing Eq. (1) and Eq. (9), it follows that:

$$
(1-b-g)=e^{-\left(v \cdot T_{c}\right) / d_{c o r r}}
$$

where $v$ is the receiver velocity, and $T_{c}$ is the time step used in the discrete time GE model (i.e., cycle time between bursts).

Comparing Eq. (2) and Eq. (5) we obtain:

$$
\frac{b}{g+b}=Q\left(\frac{\overline{S N R}-S N R_{t h}}{\sigma_{l}}\right)
$$

Using Eq. (12) and Eq. (13), the GE parameters $b$ and $g$ are easily determined. Note that the ratio $b /(g+b)$ does not depend on the dynamics of the channel. It is also intuitively clear that the GE channel become closer to being memoryless for larger $T_{c}$ or $v$, and for smaller $d_{c o r r}$.

For the correlated GE channel, the probability distribution for being in the bad state $d$ times out of $n, 0 \leq d \leq n$, is given by:

$$
\mathrm{P}_{n}(d)=\left\{\begin{array}{cc}
P^{\infty}(G)(1-b)^{n-1} & d=0 \\
P^{\infty}(G)\left(\mathrm{P}_{n}(d \mid G G)+\mathrm{P}_{n}(d \mid G B)\right) & \\
+P^{\infty}(B)\left(\mathrm{P}_{n}(d \mid B G)+\mathrm{P}_{n}(d \mid B B)\right) & 1 \leq d<n \\
P^{\infty}(B)(1-g)^{n-1} & d=n
\end{array}\right.
$$

where $\mathrm{P}_{n}(d \mid G G)$ is the conditional probability of being $d$ times in the bad state out of $n$, conditioned on being in the good state both the first and the last instants of time. The other conditional probabilities are defined accordingly. They can be computed as (for details and demonstration, interested readers 


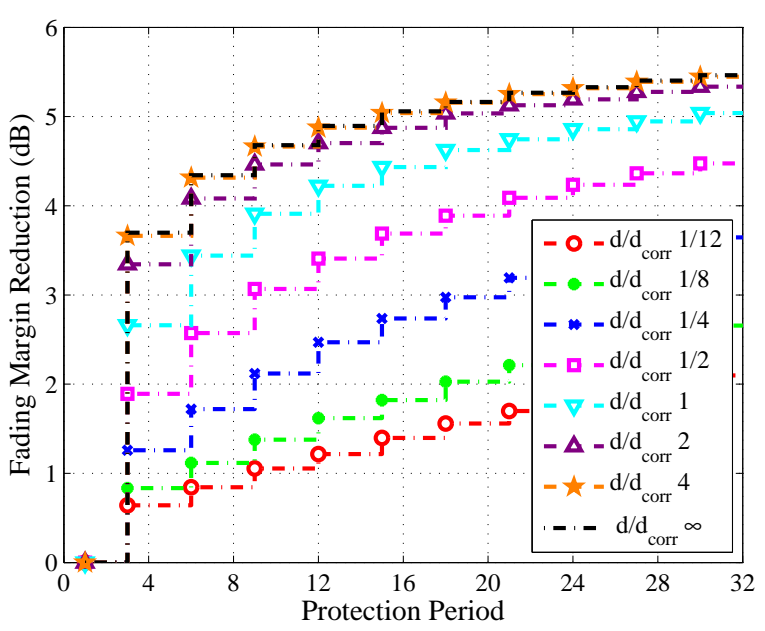

Fig. 6. Shadowing fading margin reduction for $95 \%$ service availability vs. Protection period in burst units. Code rate 2/3. Correlated shadowing: $\sigma_{l}=5.5 \mathrm{~dB}$.

are referred to [16]):

$$
\begin{aligned}
& \mathrm{P}_{n}(d \mid G G)=\sum_{i=2}^{\min (d+1, n-d)}\left(\begin{array}{c}
n-d-1 \\
i-1
\end{array}\right)\left(\begin{array}{c}
d-1 \\
i-2
\end{array}\right) \\
& \cdot(1-b)^{n-d-i} b^{i-1}(1-g)^{d-i+1} g^{i-1} \text {. } \\
& \mathrm{P}_{n}(d \mid G B)=\sum_{i=1}^{\min (d, n-d)}\left(\begin{array}{c}
n-d-1 \\
i-1
\end{array}\right)\left(\begin{array}{c}
d-1 \\
i-1
\end{array}\right) \\
& \cdot(1-b)^{n-d-i} b^{i}(1-g)^{d-i} g^{i-1} \text {. } \\
& \mathrm{P}_{n}(d \mid B G)=\sum_{i=1}^{\min (d, n-d)}\left(\begin{array}{c}
n-d-1 \\
i-1
\end{array}\right)\left(\begin{array}{c}
d-1 \\
i-1
\end{array}\right) \\
& \cdot(1-b)^{n-d-i} b^{i-1}(1-g)^{d-i} g^{i} . \\
& \mathrm{P}_{n}(d \mid B B)=\sum_{i=2}^{\min (d, n-d+1)}\left(\begin{array}{c}
n-d-1 \\
i-2
\end{array}\right)\left(\begin{array}{c}
d-1 \\
i-1
\end{array}\right) \\
& \cdot(1-b)^{n-d-i+1} b^{i-1}(1-g)^{d-i} g^{i-1} \text {. }
\end{aligned}
$$

\section{B. Analytical Results}

Fig. 6 shows the fading margin reduction achieved with inter-burst UL-FEC with a code rate $2 / 3$ for the case of correlated shadowing $\left(\sigma_{l} 5.5 \mathrm{~dB}\right)$, as a function of the interleaving depth for different $\left(v \cdot T_{c}\right) / d_{\text {corr }}$ ratios. The service availability is $95 \%$. We can see how the reduction decreases when the correlation between consecutive bursts increases.

It should be noted that there is no gain by performing an inter-burst UL-FEC protection of the transmission for fully static reception conditions (i.e., $\left(v \cdot T_{c}\right) / d_{c o r r}=0$ ), as users either receive all content or nothing.

\section{VALIDATION RESULTS}

\section{A. DVB-H Results}

Fig. 7(a) compares the theoretical fading margin results shown in Fig. 5 for uncorrelated shadowing (dashed lines) with laboratory measurement results for DVB-H (solid lines). The
DVB-H transmission mode employed in the measurements is: FFT size $8 \mathrm{~K}$, guard interval $1 / 4$, modulation 16-QAM and code rate 1/2; and for an ideal inter-burst UL-FEC code in the upper link or application layers (burst size is $2 \mathrm{Mb}$ ). The channel model employed is the typical urban 6-tap TU6 channel model for $10 \mathrm{~Hz}$ Doppler $(18 \mathrm{~km} / \mathrm{h}$ at $600 \mathrm{MHz})$. This channel model is representative for DVB-H vehicular reception [2], and it includes the time variant small-scale fluctuations of the received signal due to receiver mobility (i.e., fast fading). The shadowing component is modeled adding to the mean SNR of every burst an uncorrelated lognormal component with $5.5 \mathrm{~dB}$ standard deviation.

In the figure, we can see that the laboratory DVB-H measurement results resemble to some extent the theoretical results (the maximum difference is about $1 \mathrm{~dB}$ ). The differences are due to the fact that fast fading is not completely eliminated at the DVB-H physical layer because there is no time interleaver [2]. Hence, the inter-burst UL-FEC code copes not only with completely erroneous bursts due to shadowing, but also with partial received bursts due to fast fading [8]. As a consequence, some parity data is employed to repair partially received bursts, reducing the protection against shadowing.

However, inter-burst UL-FEC in DVB-H also reduces the fading margin required to cope with fast fading, and this reduction can even exceed the potential reduction of the fading margin due to shadowing. This occurs for high code rates, where the percentage of completely lost bursts that can be recovered is rather small (e.g., for a code rate 7/8 only one out of eight bursts can be repaired if the remaining seven bursts are received without errors), and when the shadowing correlation between consecutive bursts is high. On the other side, for robust code rates such as $1 / 2$, the potential reduction of the fading margin of only due to shadowing inter-burst UL-FEC is higher than the fading margin reduction obtained in DVB-H due to both fast fading and shadowing. The difference is larger for shorter interleaving depths, as the influence of receiving partially a burst is more significant.

\section{B. DVB-SH Results}

Fig. 7(b) compares the theoretical fading margin results shown in Fig. 5 for uncorrelated shadowing (dashed lines) with laboratory measurement results for DVB-SH (solid lines). The DVB-SH transmission mode employed in the measurements is: FFT size $2 \mathrm{~K}$, guard interval $1 / 4$, modulation QPSK, code rate $1 / 3$, and for a time interleaving depth of $210 \mathrm{~ms}$ at the physical layer (short uniform time interleaving profile), and MPE-iFEC with sliding Reed-Solomon encoding at the link layer (burst size is $2 \mathrm{Mb}$ ). The channel model employed is TU6 channel model for $40 \mathrm{~Hz}$ Doppler with uncorrelated shadowing with standard deviation $8 \mathrm{~dB}$.

In the figure, we can see that the laboratory DVB-SH measurement results coincide with the theoretical results. The reason is that the assumption that the fast fading is completely eliminated at the physical layer holds for DVB-SH. In the figure, we can also see that there are values for the protection period where the fading margin reduction is maximized. The reason is that the percentage of bursts that can be recovered 


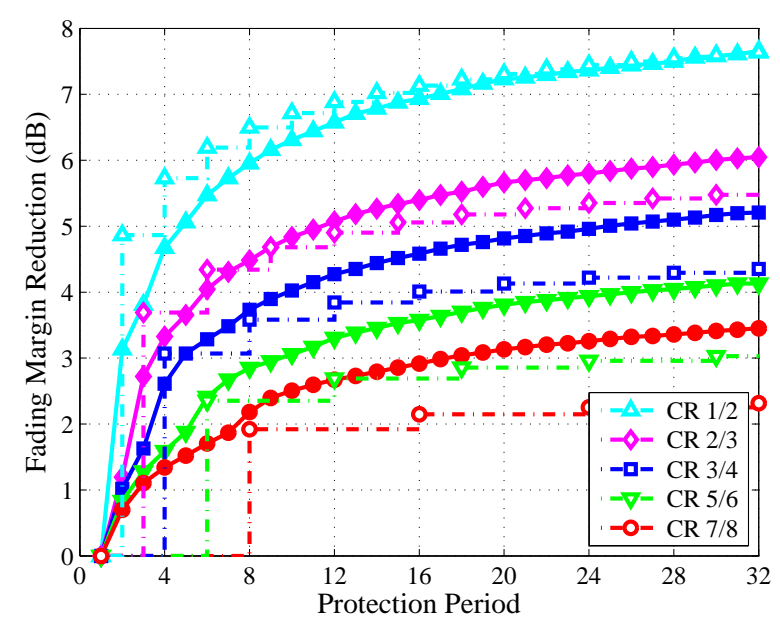

(a) DVB-H results. $\sigma_{l}=5.5 \mathrm{~dB}$.

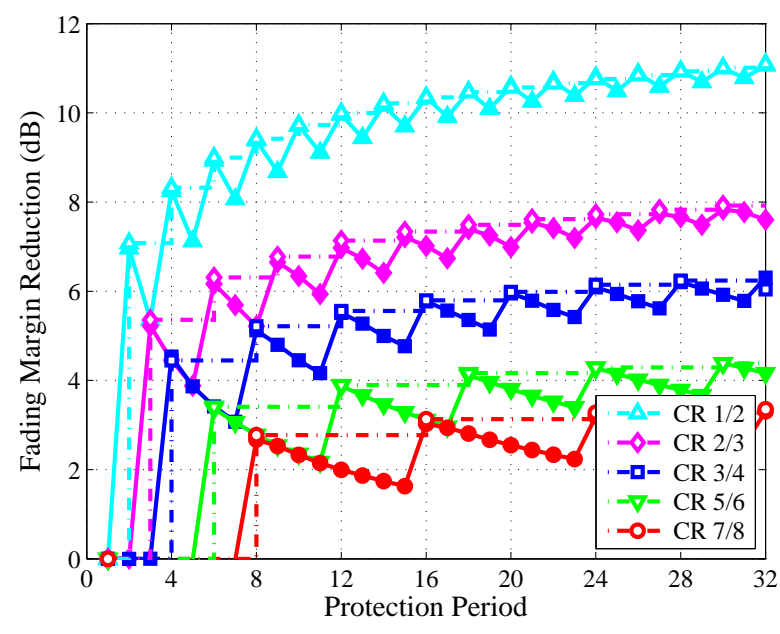

(b) DVB-SH results. $\sigma_{l}=8 \mathrm{~dB}$.

Fig. 7. Fading margin reduction for $95 \%$ service availability vs. Protection period in bursts units. Uncorrelated shadowing: $d_{c o r r}=0 \mathrm{~m}$.

is maximum (and equal to the proportion of parity data transmitted) for values of the protection period that are multiple of $n$, being $k / n$ the code rate.

\section{Discussion File DownloAd SERVICES}

File download services consist on the delivery of a finite amount of data that is stored into the terminals as a file. Typically, an error-free reception of the files is required, as even a single bit error can corrupt the whole file and make it useless for the receiver. Hence, file delivery in a generic timesliced mobile broadcast system without inter-burst UL-FEC implies that all bursts where the file is partitioned must be correctly received. Large files spanning several bursts are thus more difficult to deliver, as it is more likely to lose at least one burst.

In the following, we extend the theoretical framework to file download services for the case of uncorrelated shadowing. The case of correlated shadowing can be straightforward derived in a similar manner than for streaming services as shown in Section IV. Interested readers are also referred to [15]. Assuming that each burst experiences uncorrelated shadowing, the probability of successfully receiving the file, denoted as file acquisition probability, $F_{a c q}$, can be computed as:

$$
F_{a c q}=\mathrm{P}_{F_{s}}(0)=g^{F_{s}},
$$

where $g$ is the coverage probability and $F_{s}$ is the file size in number of bursts. We can see that the acquisition probability decreases exponentially with the file size. In lossy environments the complete file may not be fully received by most users during the initial file transmission, since some bursts may be lost. In these cases one common approach is to repeatedly transmit the file in a carousel.

If the file is transmitted $n$ times, the file acquisition probability becomes:

$$
F_{a c q}=\left(1-b^{n}\right)^{F_{s}}
$$

where $b$ is the outage probability. The problem of transmitting the file in a carousel with repetitions is that users may receive duplicate packets which are useless. If a user misses a single packet, he must wait until that specific packet is retransmitted and correctly received, discarding in the meantime packets already received.

In contrast, with inter-burst UL-FEC if a single FEC code word is applied over the entire file all source and repair packets are useful. It makes no difference which packets are received and terminals never receive duplicate data. The file acquisition probability can be computed as:

$$
F_{a c q}=\sum_{i=0}^{P P-F_{s}} \mathrm{P}_{P P}(i),
$$

where $P P=F_{s} / C R$ the total number of source and repair bursts transmitted.

Fig. 8 shows the file acquisition probability as a function of the coverage probability for different file sizes for several delivery cases. In particular, it is shown the reference case without inter-burst UL-FEC and without retransmissions, the case with one file retransmission, and the case with interburst UL-FEC with $1 / 2$ code rate. Fading margin values can be obtained in a similar manner than for streaming services as explained in Section II, but using the file acquisition probability instead of the service availability.

In Fig. 8(a) we can see that without inter-burst UL-FEC and without repetitions an efficient file delivery is only possible within the service area where the coverage probability is close to one. Hence, for this delivery case larger fading margins are required than for conventional streaming services.

When retransmitting the file once, the acquisition probability increases, see Fig. 8(b). However, the performance is still poor for large file sizes and/or for not so high coverage probabilities. Compared to retransmissions, inter-burst UL-FEC results in a much more efficient delivery of files because all correctly received packets are useful to the receivers. The gain can be translated into a reduction of the transmission time, being possible to deliver more content (files) with the same bandwidth, or into a reduction of the required fading margin. 


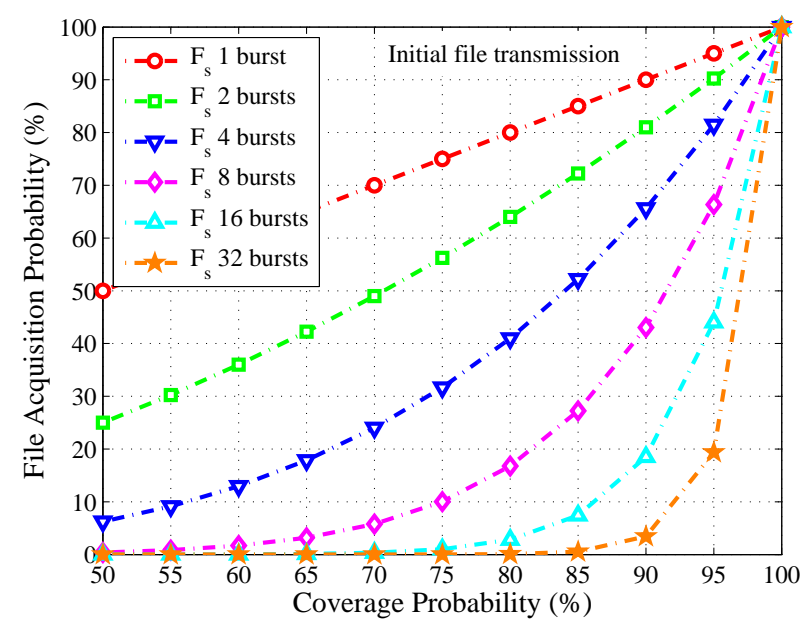

(a) Initial File Transmission.

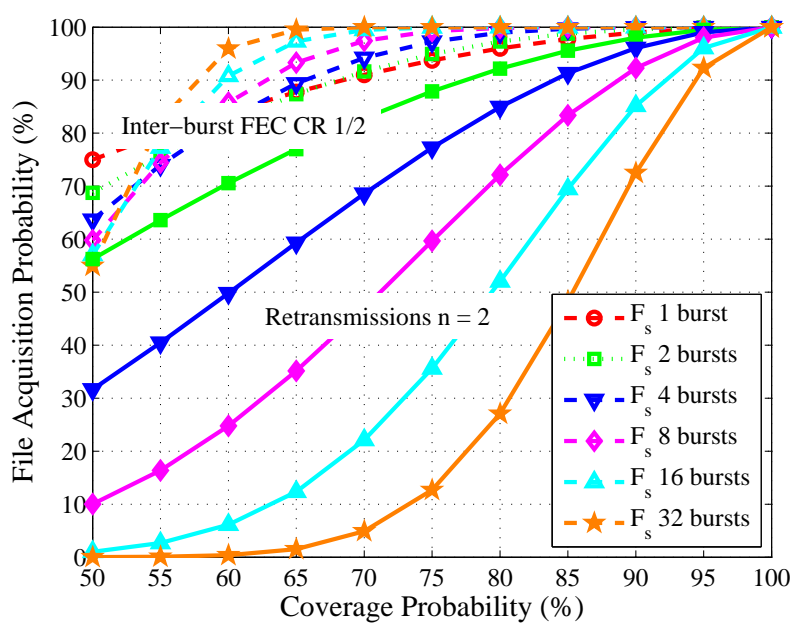

(b) Inter-burst UL-FEC $C R \quad 1 / 2$ vs. One File Retransmission.

Fig. 8. File acquisition probability vs. Coverage probability. File size in bursts units.

The effect of correlated shadowing when inter-burst UL-FEC is employed is the same as for streaming services, see Section IV. That is, the performance (acquisition probability) decreases when the correlation increases. File retransmissions also suffer a similar degradation. However, this is not true for the initial file transmission without inter-burst UL-FEC, as in this case the acquisition probability increases with the correlation towards the coverage probability.

\section{CONCLUSIONS}

In this paper we have analytically evaluated the potential reduction of the required fading margin to cope with shadowing that can be achieved with inter-burst UL-FEC in a generic time-sliced terrestrial mobile broadcast system. Results have been validated with DVB-H/SH laboratory measurements.

Inter-burst UL-FEC exploits the time diversity of the mobile channel to recover from temporary losses, increasing the service availability compared to the coverage probability. Hence, for a target service availability level, the required coverage probability is reduced, which yields a lower shadowing fading margin. The gain increases for more robust code rates, for longer interleaving and for more demanding service availabilities, and it is directly proportional to the shadowing standard deviation. The potential gain is very significant even for short interleaving depths for robust code rates such as $1 / 2$ or $2 / 3$. But the gain depends on the statistical correlation of the shadowing between consecutive bursts. The lower the correlation, the higher the gain. For a given scenario and transmission configuration, the gain depends on the user velocity. Inter-burst UL-FEC is thus especially suited for vehicular users due to their high speeds, whereas lower gains can be expected for pedestrian users due to their reduced mobility. In any case, very important gains are feasible for background services where a relatively large latency is not an issue, as it is possible to encode large amounts of bursts jointly, or to reduce the correlation level between reception conditions of consecutive bursts by increasing the cycle time between bursts. File download services fall in this category of services, and they can in general fully exploit the benefits of interburst UL-FEC. On the other hand, for streaming services it is necessary to establish an adequate trade-off between the protection provided by inter-burst UL-FEC and the latency introduced in the system, as it may compromise the user experience.

\section{REFERENCES}

[1] S. R. Saunders, "Antennas and Propagation for Wireless Communication Systems," Wiley, 2003.

[2] G. Faria, J. A. Henriksson, E. Stare, and P. Talmola, "DVB-H: Digital Broadcast Services to Handheld Devices," Proc. of the IEEE, vol. 94, no. 1, Jan. 2006, pp. 194-209.

[3] P. Kelley and C. Rigal, "DVB-SH - Mobile Digital TV in S band," $E B U$ Technical Review, July 2007.

[4] D. Gómez-Barquero, et al., "Base Band inter-frame FEC (BB-iFEC) for Next Generation Handheld DVB-NGH," Proc. IEEE BMSB, Nürnberg, Germany, 2011.

[5] A. Shokrollahi, "Raptor Codes," IEEE Trans. on Information Theory, vol. 52, no. 6, pp. 2251-2567, June 2006.

[6] ETSI TS 102584 v1.2.2, DVB bluebook A120, "Digital Video Broadcasting (DVB); DVB-SH Implementation Guidelines," May 2011.

[7] B. Sayadi, Y. Leprovost, S. Kerboeuf, M. L. Alberi-Morel, and L. Roullet, "MPE-IFEC: An Enhanced Burst Error Protection for DVB-SH Systems," Bell Labs Techn. J., vol. 14, no. 1, pp. 25-40, May 2009.

[8] D. Gómez-Barquero, D. Gozálvez, and N. Cardona, "Application Layer FEC for Mobile TV Delivery in IP Datacast over DVB-H Systems," IEEE Trans. on Broadcasting, vol. 55, no. 2, pp. 396-406, June 2009.

[9] L. Roullet, D. Gómez-Barquero, C. Hellge, L. Ottavj, and T. Stockhammer, "Digital Video Broadcasting (DVB); Upper Layer Forward Error Correction in DVB," ETSI TR 102 993, DVB bluebook A148, March 2010.

[10] J.-J. Delmas and P. Bretillon, "Mobile Broadcast Technologies - Link Budgets," BMCO forum white paper, Feb. 2009.

[11] M. Gudmunson, "Correlation Model for Shadow Fading in Mobile Radio Systems," Electronic Letters, vol. 37, no. 23, pp. 2145-2146, Nov. 1991.

[12] D. Gómez-Barquero, P. Unger, T. Kürner, and N. Cardona, "Coverage Estimation for Multiburst FEC Mobile TV Services in DVB-H Systems," IEEE Trans. on Vehicular Technology, vol. 59, no. 7, pp. 3491-3500, Sept. 2010.

[13] E. N. Gilbert, "Capacity of a Burst-Noise Channel," Bell System Technical Journal, vol. 39, pp. 1253-1265, Sept. 1960.

[14] E. O. Elliott, "Estimates of Error Rates for Codes on Burst-Noise Channels," Bell System Technical Journal, vol. 42, pp. 1977.1997, Sept. 1963. 
[15] L. R. Wilhelmsson, "Evaluating the Performance of Raptor Codes for DVB-H by using the Gilbert-Elliott Channel," Proc. IEEE VTC Fall, Baltimore, USA, 2007.

[16] L. R. Wilhelmsson and L. B. Milstein, "On the Effect of Imperfect Interleaving for the GilbertElliott Channel," IEEE Trans. on Communications, vol. 47, no. 5, pp. 681-688, May 1999.

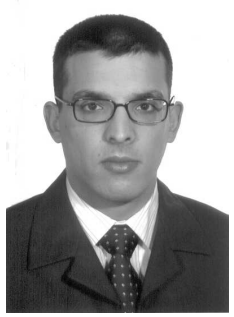

David Gómez-Barquero received a double M.Sc. degree in Telecommunications engineering from the Universidad Politécnica de Valencia (UPV), Spain, and the University of Gävle, Sweden, in 2004; and a Ph.D. in Telecommunications from UPV in 2009. During his doctoral studies he was a guest researcher at the Royal Institute of Technology, Sweden, the University of Turku, Finland, and the Technical University of Braunschweig, Germany. He also did an internship at Ericsson Eurolab, Aachen, Germany.

He is a Senior Researcher at the Mobile Communications Group of the Institute of Telecommunications and Multimedia Applications (iTEAM) of the UPV, where he leads a research group working on multimedia broadcasting in general, and in particular in the optimization of DVB (Digital Video Broadcasting) and 3GPP MBMS (Multimedia Broadcast Multicast Services) systems.

He chaired the special interest group on hybrid cellular and broadcasting networks in the European COST2100 cooperation action, and co-edited the DVB bluebook on upper layer forward error correction as an invited expert. Currently, he is is a post-doc guest researcher at the HHI Fraunhofer Research Institute for Telecommunications in Berlin, Germany, and he is actively participating in the standardization process of the next generation mobile broadcasting standard DVB-NGH, and in the validation of the second generation digital terrestrial TV system DVB-T2.

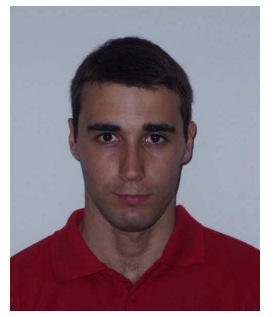

David Gozálvez received his M.Sc. degree in Telecommunications engineering from the Universidad Politécnica de Valencia (UPV), Spain, in 2007. He was the recipient of the Cátedra Telefónica prize for his Master Thesis in the same year. Currently he holds a PhD student Grant from the Spanish Government to research on transmission optimization of DVB broadcasting systems. In 2008 he undertook an internship in NOMOR research (Munich, Germany) and in 2009 and 2010 he was a guest researcher in the University of Turku (Finalnd). David Gozálvez has been an active participant in the standardization process of DVB-NGH inside the CCI (Constellation, coding and interleaving) and MIMO working groups.

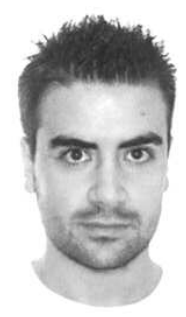

Pedro F. Gómez received his M.Sc. degree in Telecommunications engineering from the Universidad Politécnica de Valencia (UPV), Spain, in 2008. His M.Sc. thesis was awarded by the Cátedra TECATEL of the UPV and the Official College of Telecommunications Engineers of Spain (COIT). Since February 2008, he is with the Mobile Communications Group of the Institute of Telecommunications and Multimedia Applications (iTEAM) of the UPV, where he is currently working as an R\&D engineer. He has participated in several R\&D projects dealing with DVB technologies, including the Spanish project FURIA (FUtura Red Integrada Audiovisual) and the European CELTIC project ENGINES. He has been an active participant in the standardization process of DVB-NGH inside the CCI (Constellation, Coding and Interleaving) and SAT (Satellite) working groups.

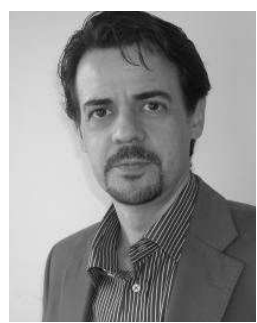

Narcís Cardona was born in Barcelona, Spain. He received a M.Sc. degree in Telecommunications engineering from the Universitat Politècnica de Catalunya, Spain, in 1990 and the Ph.D. in Telecommunications from the Universidad Politécnica de Valencia (UPV), Spain, in 1995. Since 1990 he is with the UPV, where presently he is Full Professor, and is in head of the Mobile Communications Group. Additionally he is Director of the Mobile Communications Master Degree, and Assistant Director of the Research Institute on Telecommunications and Multimedia Applications (iTEAM). Prof. Cardona has led several National research projects and has participated in some European projects, Networks of Excellence and other research forums, always in Mobile Communications aspects. At European level, he has been Vice-Chairman of the COST273 Action, and he is currently in charge of the WG3 of COST2100 in the area of Radio Access Networks. He also chaired the 3rd International Conference on Wireless Communications Systems (ISWCS'06). His current research interests include Mobile Channel Characterisation; Planning and Optimisation Tools for Cellular Systems, RRM Techniques applied to Personal Communications and Broadcast Cellular Hybrid Networks. 